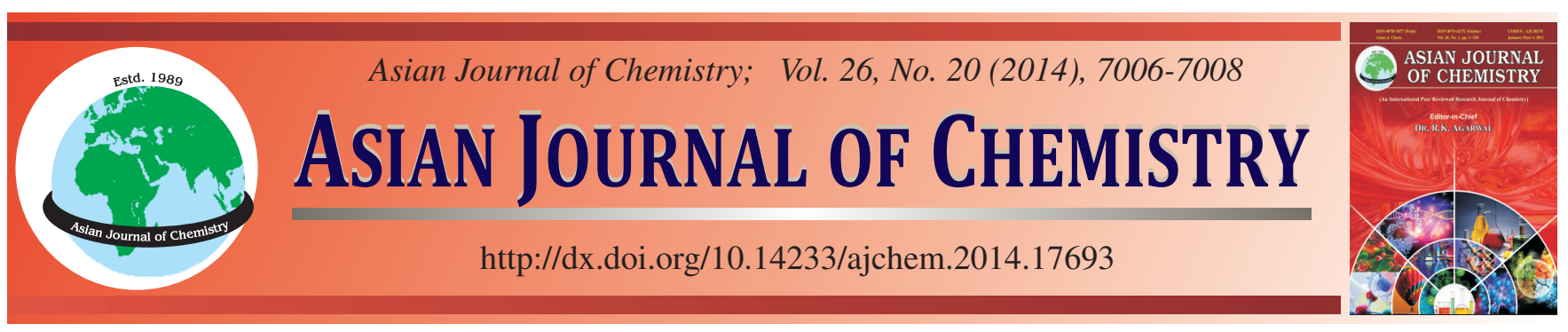

\title{
Spinacia oleracea Aqueous Extract Mediated Green Route for Synthesis of Silver Nanoparticles
}

\author{
Sobhy M. Yakout ${ }^{1,2, *}$ and Ashraf A. Mostafa ${ }^{3}$
}

${ }^{1}$ Biochemistry Department, College of Science, King Saud University, Riyadh, Kingdom of Saudi Arabia

${ }^{2}$ Atomic Energy Authority, Hot Laboratories Centre, Cairo 13759, Egypt

${ }^{3}$ Department of Botany and Microbiology, Collage of Science, King Saud University, Riyadh, Kingdom of Saudi Arabia

*Corresponding author: Fax: +966 14675931; Tel: +966 558448693; E-mail: sobhy.yakout@ gmail.com

\begin{abstract}
Metallic nanoparticles have received great attention from many scientists due its intensive applications. They are usually prepared by wet chemical methods, where the chemicals used are quite often flammable and toxic. This article gives an environmentally friendly and rapid method for synthesis of silver nanoparticles using water extract of Spinacia oleracea. Silver nanoparticles formation is proved by the presence of signatory brown color of the solution and UV-absorption peaks at $420 \mathrm{~nm}$. This method can be described as pure "green chemistry" process since no any additional synthetic chemicals are used. The phytochemicals in the planet water extract aided as effective reducing and capping agent. This study shows new opportunities in silver nanoparticles preparation.
\end{abstract}

Keywords: Silver nanoparticles, Spinacia oleracea, UV-visible.

\section{INTRODUCTION}

There is great growth in the metal nanoparticles (MNPs) fabrication ${ }^{1}$. They are widely applied in the field of science and technology. Recently, silver nanoparticles (AgNPs ) have drawn thorough attention because of their low-cost and unique properties which lead to great applications in industrial fields ${ }^{2-4}$. Currently, a large number of physicochemical and biological methods used to synthesize of MNPs ${ }^{5}$. To overcome the toxicity problem in synthesis, environmentally green methods have a major role for nanoparticles production ${ }^{6}$. Numerous methods have been used for the green synthesis of NPs using microorganisms, micro-fluids and plant extracts ${ }^{7}$. Using either microorganisms or plant extracts have researchers attention. It is an economic, simple and eco-friendly method ${ }^{8}$. Using microorganisms ${ }^{9}$ is time consuming for microorganisms maintenance while plant extract need less time. Plant extracts are readily available, easy to handle, cost effective, and have been well explored for the green biosynthesis of MNPs ${ }^{10}$. Furthermore, the biologically active molecules in the plant extract act as functionalizing ligands, making these nanoparticles more appropriate for biomedical requests ${ }^{11}$.

Spinach (Spinacia oleracea) is an edible flowering plant in the family of Amaranthaceae. It is one of the most important antioxidative vegetables, usually consumed after boiling either fresh or frozen leaves. Freshly cut spinach leaves contain approximately $1,000 \mathrm{mg}$ of total flavonoids per kilogram. The possible presence of flavonoid-like compounds in spinach was first reported ${ }^{2}$ in 1943, but nearly after 20 years before the structure of the flavonol isolated from spinach leaves was established as patuletin $\left(3,5,7,3^{\prime}, 4^{\prime}\right.$-penthahydroxy-6-methoxyflavone) and the presence of spinacetin was confirmed ${ }^{3}$. In addition, the existence of several flavonol glycosides in a methanolic extract of spinach leaves was reported ${ }^{4,5}$. The occurrence of atleast 10 flavonoid glycosides has now been reported in spinach.

Hence, the present study was designed to prepare silver nanoparticles using bio-green method with aqueous extract of Spinacia oleracea. The formed AgNPs were characterized using UV-visible spectroscopy. Understanding of the in situ particle stabilization mechanism are reported in this study.

\section{EXPERIMENTAL}

All chemicals and reagents used in the present study are of high purity and are obtained from Sigma and Merck. All glasswares were washed with nitric acid, distilled water and dried in oven. Spinacia oleracea were collected from were collected from local market of Riyadh, Saudi Arabia.

Preparation of Spinacia oleracea extract: The fresh plant leaves (Fig. 1) were washed several times with running tap water and then with distilled water. $20 \mathrm{~g}$ of dried leaves were weighed and boiled for $15 \mathrm{~min}$ in $100 \mathrm{~mL}$ Milli-Q water and then the extracts were filtered through Whatmann filter paper No. 1 . The filtered extract was stored in refrigerator at $4{ }^{\circ} \mathrm{C}$. 


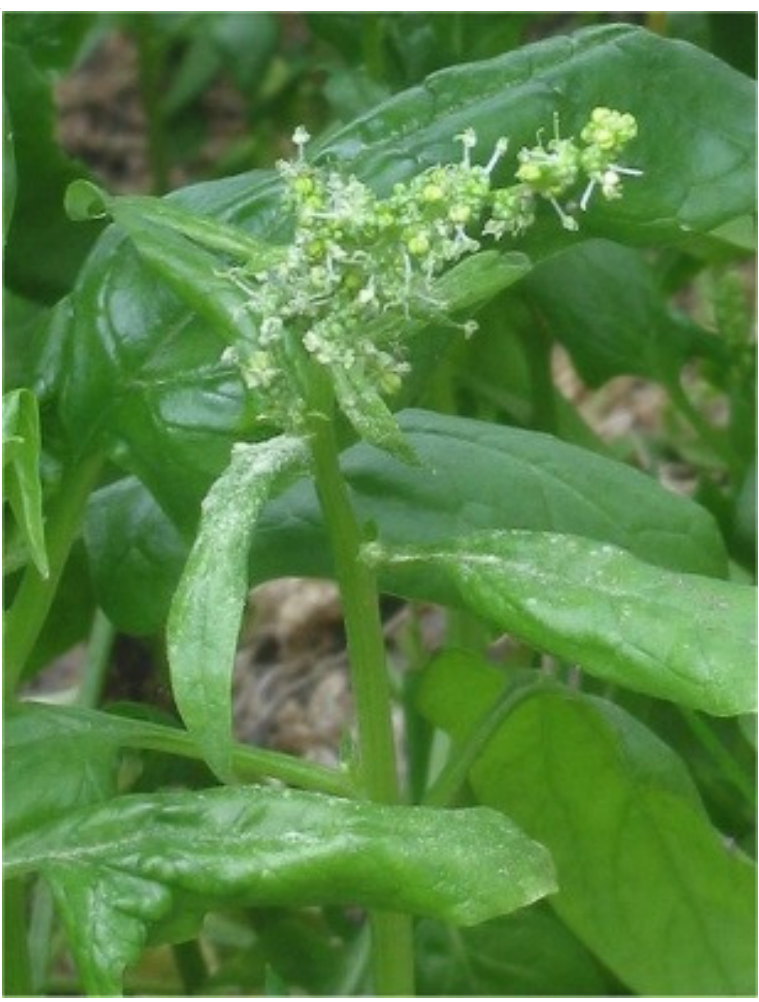

Fig. 1. Spinacia oleracea leaves

Bio-green synthesis of silver nanoparticles: The synthesis of nanoparticles by bio-green method was carried by adding $10 \mathrm{~mL}$ of aqueous extract of Spinacia oleracea (Fig. 2a) to $50 \mathrm{~mL}$ of $1 \mathrm{mM}$ silver nitrate solution and kept for incubation at room temperature for $2 \mathrm{~h}$. The overall reaction process was carried out in dark to avoid unnecessary photochemical reactions. The color change of the silver nitrate solution from colourless to brownish yellow was observed by naked eye (Fig. 2b) and the bio reduced sample component was confirmed by UV-visible spectroscopy. The obtained Spinacia olerace $\mathrm{AgNPs}$ were purified through repeated centrifugation at 11,500 rpm for $20 \mathrm{~min}$ and washed with distilled water. The Spinacia oleracea AgNPs were collected and redispersed in deionized water for characterization.
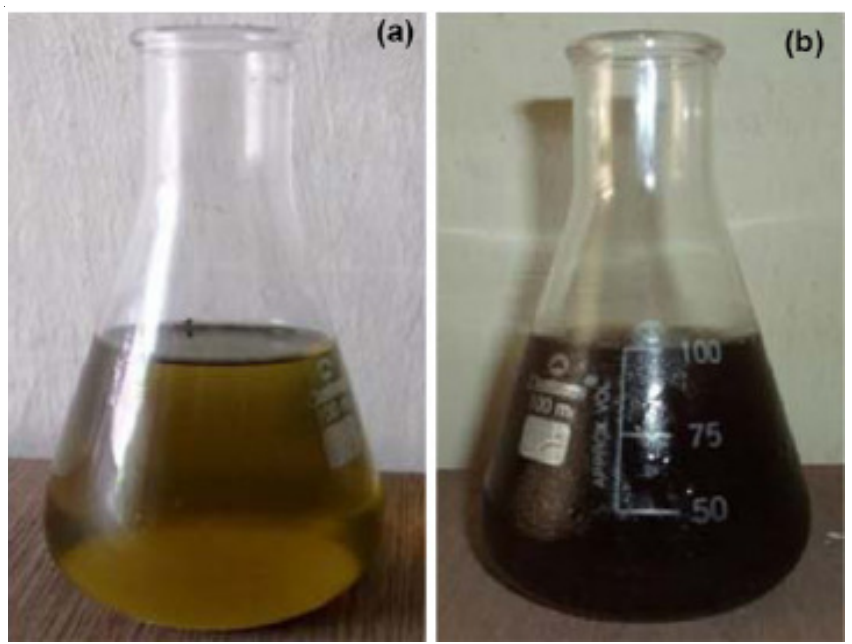

Fig. 2. Biosynthesis of AgNP susing Spinacia oleracea leaves visible observations: (a) plant extract (b) silver nanoparticles
UV-visible spectroscopy: Formation of AgNPs was monitored by measuring the UV-visible spectra of the respective solutions after diluting it 20 times with deionized water. The spectrum was measured by using UV-1700 Shimadzu UVvisible spectrophotometer from 300 to $600 \mathrm{~nm}$. Deionized water was used as the blank.

\section{RESULTS AND DISCUSSION}

Preparation and characterization of silver nanoparticle: AgNPs formation of silver nitrate and Spinacia oleracea water extract can be easily seen by the color change of the mixture from green to yellowish brown color which is specific to AgNPs as seen in Fig. 2. This color arises from excitation of surface plasmon vibrations in the metal nanoparticles ${ }^{12,13}$ due to collective oscillation of free electrons present in the reduced $\mathrm{AgNPs}^{14}$. Such visual observations on a change color due to the synthesis of gold nanoparticles have been reported earlier ${ }^{15}$.

Due to, surface plasmon resonance (SPR) phenomenon, UV-visible spectroscopy is is used in characterization of metallic nanoparticles ${ }^{16}$. Fig. 3 shows the UV-visible absorption spectra of the Ag nanoparticles at different time intervals (10, 20, 30, 60 and $90 \mathrm{~min}$ ). The obtained absorption peak is centered on $420 \mathrm{~nm}$. Typical AgNPs having $\lambda_{\max }$ values in the visible range of $400-500 \mathrm{~nm}^{17}$. This observation obviously shows the successful reduction of Ag using Spinacia oleracea water extract. Surface Plasmon peak observed confirms the effect of aqueous Spinacia oleracea extract in reducing $\mathrm{Ag}^{+}$ ions to $\mathrm{Ag}$ nanoparticles. The formation of $\mathrm{Ag}$-nanoparticles was found to rise with reaction time from 10 to $90 \mathrm{~min}$. The broad spectra formed at short reaction time due to the presence of particles with a wide size distribution. The slight shift in the peak to shorter wavelength with time observed may be attributed to slight modification in the size and shape of nanoparticles ${ }^{18}$. As the time increases, the absorption peak becomes sharp. This due tothe formation of the poly dispersed Agnanoparticles originally that become mono-dispersed spherical nanoparticles with increasing reaction time. Therefore, mixing time is an important factor in determining the size distribution of Ag-nanoparticles inferred at room temperature.

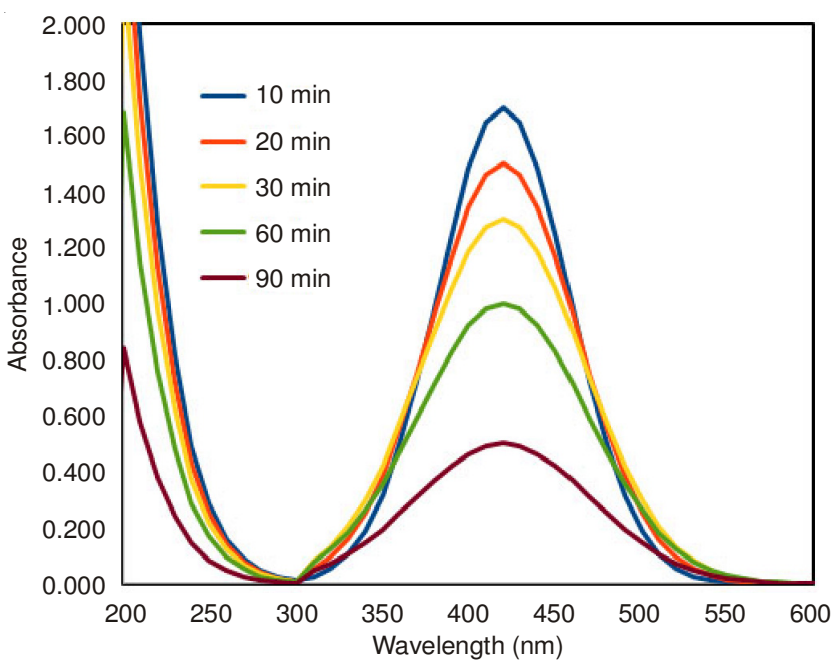

Fig. 3. UV-visible spectra of AgNPs synthesized by Spinacia oleracea leaves at different time intervals 
Mechanism of the formation of silver nanoparticle: Spinacia oleracea water extract, containing different phytochemicals, including flavonoids, exhibit antioxidative, antiproliferative that considered as active components and have antiinflammatory properties in biological systems. Phenolic acids are other large family of secondary metabolites having hydroxyl benzoic or hydroxyl cinnamic structures. They possess hydroxyl and carbonyl groups which are able to bind to metals ${ }^{19}$. Phenolic compounds may inactivate ions by chelating. Due to the high nucleophilic character of the aromatic rings rather than to specify chelating groups in the structure of these molecules.

\section{Conclusion}

We introduce a simple, fast and economical biological procedure to synthesize silver nanoparticles using Spinacia oleracea water extract as reducing and capping agents. UVvisible techniques confirm the reduction of silver ions, exposed to this plant extracts, to silver nanoparticles. The presence of phenolic compounds may be the key factors for the formation of silver nanoparticles. Biological synthesized AgNPs could be of immense use in the medical field for their efficient antimicrobial function. Moreover, this study can be extended to prepare similar other metal nanoparticles also.

\section{ACKNOWLEDGEMENTS}

This project was supported by King Saud University, Deanship of Scientific Research, College of Science Research Centre.

\section{REFERENCES}

1. K. Balantrapu and D. Goia, J. Mater. Res., 24, 2828 (2009).

2. K. Bhatte, P. Tambade, K. Dhake and B. Bhanage, Catal. Commun., 11, 1233 (2010).

3. J. Musarrat, S. Dwivedi, B.R. Singh, A.A. Al-Khedhairy, A. Azam and A. Naqvi, Bioresour. Technol., 101, 8772 (2011).

4. R. Kosmala, R. Wright, Q. Zhang and M.P. Kirby, Mater. Chem. Phys., 129, 1075 (2011).

5. M. Mahdavi, M. Ahmad, M. Haron, F. Namvar, B. Nadi, M. Rahman and J. Amin, Molecules, 18, 7533 (2013).

6. F.A.A. Rajathi, C. Parthiban, V. Ganesh Kumar and P. Anantharaman, Spectrochim. Acta A, 99, 166 (2012).

7. K. Shameli, M. Ahmad, P. Shabanzadeh, A. Zamanian, P. Sangpour, Y. Abdollahi and Z. Mohsen, Int. J. Nanomed., 7, 5603 (2012).

8. P. Raveendran, J. Fu and S. Wallen, J. Am. Chem. Soc., 125, 13940 (2003).

9. K.N. Thakkar, S.S. Mhatre and R.Y. Parikh, Nanomedicine, 6, 257 (2010).

10. M. Khan, M. Khan, S.F. Adil, M.N. Tahir, W. Tremel, H.Z. Alkhathlan, A. Al-Warthan and M.R.H. Siddiqui, Int. J. Nanomed., 8, 1507 (2013).

11. S. Azizi, F. Namvar, M. Mahdavi, M. Ahmad and R. Mohamad, Materials, 6, 5942 (2013).

12. A.A. El-Khatib, Environ. Pollut.,124, 419 (2003).

13. S. Ishtiaq, S. Saeed-ul-Hassan, U. Niaz, M.A. Saeed, Pak. J. Pharm. Sci., 25, 589 (2012)

14. M.F. Zayed, W.H. Eisa and A.A. Shabaka, Spectrochim. Acta A, 98, 423 (2012).

15. A. Ahmad, P. Mukherjee, S. Senapati, D. Mandal, M.I. Khan, R. Kumar and M. Sastry, Colloids Surf. B, 28, 313 (2003).

16. S.L. Smitha, D. Philip and K.G. Gopchandran, Spectrochim. Acta A, 74, 735 (2009).

17. M. Sastry, K.S. Mayya and K. Bandyopadhyay, Colloids Surf. A, 127, 221 (1997)

18. D. Raghunandan, B.D. Mahesh, S. Basavaraja, S.D. Balaji, S.Y. Manjunath and A. Venkataraman, J. Nanopart. Res., 13, 2021 (2011).

19. S. Ashok Kumar, S. Ravi, V. Kathiravan, S. Velmurugan and S.D. Balaji, Spectrochim. Acta A, 121, 88 (2014). 